\title{
Genomic pathology: a disruptive innovation
}

"...genomic pathology [is] founded on the premise that sequencing the human genome is rapidly becoming so routine and inexpensive that it will inevitably find its way into fundamental aspects of healthcare - not only in disease diagnosis and management, but also disease prevention, risk mitigation and health maintenance."

KEYWORDS: disruptive innovation " genomic pathology " next-generation sequencing whole-genome analysis

\section{Disruptive innovation}

"Disruptive innovation describes a process by which a product or service takes root initially in simple applications at the bottom of a market and then relentlessly moves 'up market', eventually displacing established competitors. An innovation that is disruptive allows a whole new population of consumers access to a product or service that was historically only accessible to consumers with a lot of money or a lot of skill. Characteristics of disruptive businesses, at least in their initial stages, can include: lower gross margins, smaller target markets, and simpler products and services that may not appear as attractive as existing solutions when compared against traditional performance metrics."

- Clayton Christenson,

Harvard Business School (MA, USA)

This special focus issue of Personalized Medicine is all about genomic pathology, a concept founded on the premise that sequencing the human genome is rapidly becoming so routine and inexpensive that it will inevitably find its way into fundamental aspects of healthcare not only in disease diagnosis and management, but also disease prevention, risk mitigation and health maintenance. The disruptive innovation that will make this possible is next-generation sequencing (NGS) and other high-throughput technologies to define in exquisite detail an individual human being's genome, transcriptome, proteome, metabolome/lipidome and microbiome. These rapidly emerging technologies are perfect examples of Christensen's paradigm [101]. In the few short years since the human genome project culminated in the first complete sequence of a human genome, the pace of technological advancement has been breathtaking. The result is that today, thousands of genomes have been sequenced, the capacity to sequence human genomes is growing exponentially, and the cost continues to plummet such that the impact of sequencing human genomes will become an insignificant factor in healthcare economics. Indeed, NGS and related high-throughput technologies will be the disruptive innovation to fully realize the promise of personalized medicine.

The parallels of NGS to Christensen's concept of disruptive innovation are striking. Today, NGS is still largely 'at the bottom' of the clinical laboratory diagnostics 'market'. It now resides mainly within the rarefied domain of large-scale genome research centers such as the Broad Institute of Harvard (MA, USA) and the Massachusetts Institute of Technology (MA, USA), the Wellcome Trust Sanger Institute (Hinxton, UK), and the Genome Sequencing Center of Washington University (MO, USA). Within this market, NGS is accessible only to 'consumers with a lot of money or a lot of skill'. But we are witnessing a great change. BGI (Shenzhen, China), formerly known as the Beijing Genomics Institute, has the capacity to sequence more than 15,000 human genomes per year. Forbes magazine has estimated that the genomics market will reach US $\$ 100$ billion over the next decade [1]. The current state of genomics has been likened to the 'early days of the internet' and we can anticipate the emergence of a number of 'killer apps' [2]. As the technology advances and the costs come down, NGS will be ready for a 'whole new

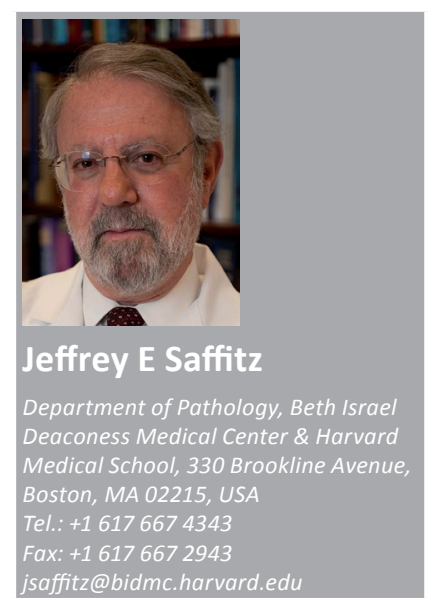

Future :
Medicine $\%$ part of 
population of consumers' - clinical laboratories and laboratory-based physicians who work in virtually every hospital in the nation and provide the bulk of objective clinical data used by treating physicians in diagnosis, evaluation and management of disease.

\section{"...next-generation sequencing and related high-throughput technologies will be the disruptive innovation to fully realize the promise of personalized medicine."}

The disruption will occur in two major ways. First, NGS will replace many current laboratory tests - not only those involving molecular/genetic testing in cancer and cytogenetics, but others such as clinical microbiology or histocompatibility. The 'value-proposition' will be realized by replacing multiple current technologies, each requiring expensive instrumentation and highly trained personnel, with a single, cost-effective technology platform that can be deployed across multiple clinical laboratory activities. Second, as more and more healthy individuals have their genomes analyzed, NGS will lead a disruptive shift in the role of clinical laboratories in healthcare by driving a radical departure from the traditional boundaries of disease diagnosis to a new frontier of highly personalized programs of disease prevention, life-style modification and risk mitigation. This oncoming change from reaction to prevention has given rise to the concept of 'primary care pathology' [3], an opportunity for laboratory-based physicians to capture the value of low-cost, high-throughput genome technologies to produce and use genetic data in precision diagnostics and proactive, individualized disease prediction and prevention.

Of course, we are still far from full implementation of NGS and related technologies in medicine. We can imagine the future potential, but we also recognize enormous obstacles that must be surmounted first. How to get there is the subject of the articles in this special focus issue. Perhaps the first step is a healthy dose of reality checking. This is the central message of the commentary by Schrijver and Galli, which tempers excitement about what might be possible with a cautious warning about promising too much too soon [4]. A critical requirement in realizing the promise of personalized medicine is physician education. Nowhere in the current state of undergraduate or postgraduate medical education is there systematic, formal training in even rudimentary aspects of high-throughput genome technologies much less their potential applications in clinical care. As described by Haspel and colleagues, this deficiency is being addressed through a national effort to create and disseminate a basic training module in genomics and personalized medicine that will be required of all pathologists-in-training [5]. Successful implementation of this program will ensure that a cadre of laboratory-based physicians has the necessary proficiency to drive the disruptive diagnostics of the future.

Bringing NGS and whole-genome analysis into everyday clinical laboratory diagnostics will be a complex undertaking requiring advances in technology, informatics and interfaces with conventional components of the medical record. As discussed by Wall and Tonellato, the first steps will involve a number of demonstration projects to test paradigms, identify and overcome roadblocks and set the stage for new strategies in clinical decision support [6]. Another critically essential goal, covered in the commentary by Wall and Tonellato, is the establishment of a 'clinical grade' database of human genome sequence variants of sufficient quality that a physician can reliably use the outcomes of whole-genome analysis in patient care. Fulfilling this goal will require a mechanism by which clinically actionable sequence variants can be defined and rigorously separated from the overwhelmingly vast amount of naturally occurring genomic variability that distinguishes one human being from another but does not necessarily contribute to disease.

\section{“...next-generation sequencing will replace many current laboratory tests - not only those involving molecular/genetic testing in cancer and cytogenetics, but others such as clinical microbiology or histocompatibility."}

Many crucial obstacles beyond the purely technical and scientific data must be addressed to bring NGS into clinical care. Among these are the need to develop a rational set of rules and regulations to oversee the various devices, instruments and information technologies required for NGS applications, and to ensure that physicians engaged in these activities have the appropriate training and expertise. This is the subject of the thoughtful review by Walcoff and Pfeifer [7]. And, as reviewed by Crawford and Aspinall there must be new business models to accommodate the new powers of NGS and related technologies [8]. Such models must account not only for the traditional diagnostic approach in disease management, but also recognize the promise of 
disease prevention and risk mitigation strategies which have the potential to save vast amounts of money but must be appropriately reimbursed if they are to succeed. Finally, this special focus issue contains insightful interviews and an editorial from experts with uncommon vision and a strong grounding in realistic expectations. Taken together, these papers provide a logical and comprehensive blueprint for the future of genomic pathology.

\section{Financial \& competing interests disclosure}

The author has no relevant affliations or financial involvement with any organization or entity with a financial interest in or financial conflict with the subject matter or materials discussed in the manuscript. This includes employment, consultancies, honoraria, stock ownership or options, expert testimony, grants or patents received or pending, or royalties.

No writing assistance was utilized in the production of this manuscript.

\section{References}

1 Herper M. The next $\$ 100$ billion technology business. 30 December Forbes (2010).

2 Quotes attributed to George Church. 24 April Newsweek (2011).

3 Tonellato PJ, Crawford JM, Boguski MS, Saffitz JE. A national agenda for the future of pathology in personalized medicine. Am. J. Clin. Pathol. 135(5), 668-672 (2011).

4 Schrijver I, Galli SJ. Between hype and hope: whole-genome sequencing in clinical medicine. Per. Med. 9(3), 243-239 (2012).
5 Haspel RL, JB Atkinson, FG Barr. TRIG on TRACK: educating pathology residents in genomic medicine. Per. Med. 9(3), 287-293 (2012).

6 Wall DP, Tonellato PJ. Deriving clinical action from whole-genomic analysis. Per. Med. 9(3), 247-252 (2012).

7 Walcoff SD, Pfeifer JD. Modernizing US regulatory and reimbursement policy to support continued innovation in genomic pathology. Per. Med. 9(3), 295-308 (2012).
8 Crawford JM, Aspinall MG. The business value and cost-effectiveness of genomic medicine. Per. Med. 9(3), 265-239 (2012).

\section{Website}

101 Key Concepts: Disruptive Innovation. www.claytonchristensen.com/disruptive innovation.html 